\title{
Relationship between insecticide resistance and $k d r$ mutations in the dengue vector Aedes aegypti in Southern China
}

\author{
Chun-Xiao Li', Phillip E Kaufman², Rui-De Xue ${ }^{3}$, Ming-Hui Zhao ${ }^{1}$, Gang Wang ${ }^{1}$, Ting Yan', Xiao-Xia Guo ${ }^{1}$, \\ Ying-Mei Zhang ${ }^{1}$, Yan-De Dong ${ }^{1}$, Dan Xing ${ }^{1}$, Heng-Duan Zhang ${ }^{1}$ and Tong-Yan Zhao ${ }^{1 *}$
}

\begin{abstract}
Background: Aedes aegypti is an important vector for dengue virus and thus has been targeted with pyrethroid insecticides in many areas of the world. As such, resistance has been detected to several of these insecticides, including in China, but the mechanisms of the resistance are not well understood in this country.

Methods: Using the World Health Organization larval mosquito bioassay, five field populations of Aedes aegypti from Southern China were characterized for their resistance to cypermethrin and cyhalothrin. RNA extraction with PCR amplification, cloning and sequencing of the sodium channel gene was followed by comparisons of susceptible and wild mosquito strains Additionally, genomic DNA was used for Allele-specific PCR (AS-PCR) genotyping of the sodium channel genes to detect S989P, V1016G and F1534C mutations and allow for correlation analysis of resistance expression for the different mutations.

Results: All wild strains expressed resistance to cypermethrin and cyhalothrin and the resistance expression between the two insecticides was highly correlated suggesting cross-resistance between these two pyrethroids. The AS-PCR technique effectively distinguished individual genotypes for all three mutations. Among the five wild strains tested, two strains carried all three mutations. Although the S989P and V1016G mutations were positively correlated to resistance expression of both pyrethroids, the F1534C mutation was negatively correlated.

Conclusions: Our methodology proved highly reliable and will aid future detection of kdr mutations. The three sodium channel mutations were common in the Ae. aegypti strains sampled from Southern China. The V1016G mutation appears to be the most important kdr mutation in Ae. aegypti strains in Southern China.
\end{abstract}

Keywords: Aedes aegypti, kdr mutation, China

\section{Background}

The mosquito Aedes aegypti (L.), a primary vector of dengue fever and dengue hemorrhagic fever, mainly occurs in tropical areas. Because there is currently no dengue vaccine, mosquito control has become the most important method of preventing transmission of these diseases [1] and insecticides are expected to remain a key component of dengue control for the foreseeable future [2]. Pyrethroids, characterized by their high toxicity

\footnotetext{
* Correspondence: tongyanzhao@126.com

'Department of Vector Biology and Control, State Key Laboratory of Pathogen and Biosecurity, Institute of Microbiology and Epidemiology, Beijing 100071, China

Full list of author information is available at the end of the article
}

to target insects and relatively few adverse effects on mammals, have been used widely in mosquito control. However, many mosquito populations have developed resistance to these compounds following repeated exposure to them $[3,4]$.

Both pyrethroids and DDT target the voltage-gated sodium channel of insect neurons and single amino acid substitutions in the sodium channel have been associated with resistance to both these insecticides [5]. This form of resistance, known as knockdown resistance $(k d r)$, has been observed in several insect species including the mosquitoes Anopheles gambiae Giles [6], An. stephensi Liston [7], Culex quinquefasciatus Say [8] and Ae. aegypti [9]. 
In many insect species, mutations related to both pyrethroid and DDT resistance largely have been located in the IIS6 region. Of these, the replacement of a Leu for a Phe in the 1014 site (Leu1014Phe), first described in a DDT resistant strain of Musca domestica L. [10], was the most common. Other substitutions in the same homologous site have been described in a number of species, such as Leu1014Ser in the mosquitoes An. gambiae [11] and Culex pipiens L. [12] and Leu1014His in the tobacco budworm Heliothis virescens (Fabricius) [13]. In Ae. aegypti, substitutions at site 1014 are unlikely, as two independent changes in the same codon would be necessary [14]. Instead, mutations in different positions have been observed in Latin American and Southeast Asian populations of Ae aegypti. So far, a number of $k d r$ mutations have been identified in Ae. aegypti (S989P, I1011M/V, V1016G/I, F1269C, F1534C) [9, 14, 15] and at least two of these are known to be related to pyrethroid resistance; 1016 (Val to Ile or Gly) and 1534 (Phe to Cys) in the IIS6 and IIIS6 segments of the Vssc gene, respectively $[9,14,16,17]$.

Ae. aegypti is present in many residential areas of Southern China. In recent years, regular monitoring has revealed that many Chinese populations of Ae. aegypti have become resistant to pyrethroid insecticides [18, 19], but the underlying mechanism responsible for this resistance is unknown. The aims of this study were to detect and identify mutations in the sodium channel gene and to determine whether any of these are linked to pyrethroid resistance in wild Ae. aegypti populations in China. We used the WHO susceptibility test to evaluate the resistance of Ae. aegypti larvae to commonly used pyrethroid insecticides and molecular assays to identify specific point mutations in the sodium channel gene of $A e$. aegypti collected from various regions in Southern China. The relationship between pyrethroid knockdown resistance and the frequencies of different $k d r$ alleles is analysed and discussed. A novel method has also been developed to detect the mutation found in Chinese Ae. aegypti field strains and to determine the role of the mutations in cypermethrin and cyhalothrin resistance in China. Determining the distribution of one of the major mechanisms underpinning $k d r$ mutations in wild mosquito populations is important to improving the effectiveness of dengue control operations.

\section{Methods}

\section{Mosquito specimens}

Larvae of Ae. aegypti were collected from five sites in Southern China in 2013: Qishui $\left(20^{\circ} 45^{\prime} 46.3^{\prime \prime} \mathrm{N}, 109^{\circ} 45^{\prime}\right.$ 35.0"E), Dongfang (19 $\left.6^{\prime} 3.8^{\prime \prime} \mathrm{N}, 108^{\circ} 35^{\prime} 8.6^{\prime \prime} \mathrm{E}\right)$, Jinghan $\left(24^{\circ} 01^{\prime} 51.5^{\prime \prime} \mathrm{N}, 97^{\circ} 53^{\prime} 15.6^{\prime \prime} \mathrm{E}\right)$, Yundang $\left(24^{\circ} 01^{\prime} 25.6^{\prime \prime} \mathrm{N}\right.$, $\left.97^{\circ} 52^{\prime} 58.9^{\prime \prime} \mathrm{E}\right)$ and Munao $\left(24^{\circ} 01^{\prime} 08.6^{\prime \prime} \mathrm{N}, 97^{\circ} 51^{\prime} 47.8^{\prime \prime} \mathrm{E}\right)$. Larvae were collected from standing water sources such as jars, bowls, tanks, tin cans and drums. No specific permissions were required to collect larvae as their collection did not involve, or affect, any endangered or protected species. Collected larvae were transported to the laboratory and colonized. Bioassays for pesticide resistance were conducted on the first $\left(\mathrm{F}_{1}\right)$ generation of larvae produced in captivity and the results compared to those of a pyrethroid-susceptible Ae. aegypti strain. The susceptible Ae. aegypti strain originated from Hainan province, China, and has been isolated in a laboratory for more than 10 years without exposure to insecticides. Larvae were flash-frozen at $-70{ }^{\circ} \mathrm{C}$ after pesticide exposure trials.

\section{Mosquito bioassay}

The resistance of larvae to two pyrethroids, cypermethrin ( $\geq 98 \%$, Sigma, USA) and cyhalothrin ( $>99 \%$, Sigma, USA), was evaluated. Thirty late 3rd and early 4th instar larvae were placed in a glass container that held $199 \mathrm{ml}$ water and $1 \mathrm{~mL}$ of an insecticide concentration using the methods outlined by the WHO [20]. All analytical grade insecticides were diluted five to seven concentrations with acetone. The serial concentrations were $0.0002,0.0005$, $0.001,0.003,0.005,0.008$ and $0.01 \mathrm{ppm}$ for the susceptible strain, Qishui strain and Dongfang strain. The serial concentrations were $0.005,0.01,0.03,0.05,0.08 \mathrm{ppm}$ for Jinghan strain and Yundang strain. The serial concentrations were $0.025,0.05,0.1,0.15$ and $0.2 \mathrm{ppm}$ for Munao strain. A control with no insecticide was done in order to control for the natural mortality of the strain tested. All experiments were repeated three times and larval mortality recorded $24 \mathrm{~h}$ after treatment. Larval mortality was determined by dividing the number of dead larvae by the total number tested. No food was provided to larvae during bioassays, which were conducted under a $14 \mathrm{~L}$ : 10D photoperiod, $75 \%$ relative humidity and temperature of $26 \pm 1{ }^{\circ} \mathrm{C}$.

$\mathrm{LC}_{50}$ values were calculated using Schoofs and Willhite's [21] probit analysis program. As a measure of resistance we calculated the resistance ratio (RR) [22], which was the ratio of the estimated $\mathrm{LC}_{50}$ of the $\mathrm{F}_{1}$ captive generation to that of larvae of the susceptible strain. In order to examine whether resistance was correlated among sampling sites, relationships between estimated $R R$ values of various insecticides were analyzed by Pearson correlation analysis. $P$-values $<0.05$ were considered statistically significant.

\section{Extraction of mosquito RNA and synthesis of mosquito CDNA}

Total RNA of 30 female mosquitoes from the susceptible laboratory strain and the five wild strains were extracted with TRIzol reagent according to the manufacturer's protocol (Invitrogen, U.S.A.). These samples came from the same generation and age classes (late 3rd and early 
4th instar) used for the resistance bioassay. The yield and purity of the extracted RNA were assessed by determining its absorbance (Abs) at 260 and $280 \mathrm{~nm}$. RNA was used only if its Abs260 nm/Abs280 nm ratio was $>1.8$. Extracted total RNA was stored at $-70{ }^{\circ} \mathrm{C}$ for later use.

Two microliters of total RNA from each sample was used to synthesize the first-strand cDNA using a oligo(dT) ${ }_{12-18}$ primer in a $20-\mu l$ reaction following the Superscript ${ }^{\mathrm{TM}}$ RT-PCR System (Invitrogen, U.S.A.). The first-strand cDNA reaction was stored at $-20{ }^{\circ} \mathrm{C}$ until required.

PCR amplification of the Ae. aegypti sodium channel gene Gene-specific primers based on the published sequence of the Ae. aegypti para-sodium channel gene (GenBank Accession No.: EU399179.1) were designed in NCBI Primer BLAST and used to amplify the sodium channel gene of specimens from each strain. The sodium channel gene is $6554 \mathrm{bp}$ in length and was amplified as 11 fragments. The primers used are shown in Table 1. The PCR reaction consisted of one cycle of $94{ }^{\circ} \mathrm{C}$ for $3 \mathrm{~min}$ and 35 cycles at $94{ }^{\circ} \mathrm{C}$ for $30 \mathrm{~s}, 46 \sim 63{ }^{\circ} \mathrm{C}$ for $30 \mathrm{~s}$ (different Tm for 11 fragments' amplification), $72{ }^{\circ} \mathrm{C}$ for $1 \mathrm{~min}$ and a final extension step at $72{ }^{\circ} \mathrm{C}$ for $7 \mathrm{~min}$.

\section{Cloning and sequencing of PCR products}

PCR products were run on a $1.5 \%$ agarose gel and purified using Wizard PCR Preps DNA Purification System (Promega, U.S.A.). Purified PCR products were ligated into pMD 18-T easy vector (Takara, Japan) and plasmids cloned into E. coli XL1-blue competent cells. Plasmids were purified using a miniBEST plasmid purification kit (Takara, Japan). At least 30 clones of each PCR product were selected. Sequencing reactions were carried out on recombinant plasmids using M13 forward and reverse primers. Sequence alignment was performed with the Clustal W algorithm [23].

\section{Comparison of sodium channel genes from susceptible and wild strains}

The amino acid and cDNA sequences of susceptible and wild strains collected in 2013 were analysed with DNAstar software [24]. Blast research was performed at http://www.ncbi.nlm.nih.gov/blast/. Sequences were aligned with ClustalX software [23] and viewed with GeneDoc software [25].

\section{Genomic DNA isolation}

Genomic DNA was isolated from $32 \sim 36$ female mosquitoes from each strain using a Universal Genomic

Table 1 The eleven pairs of primers used to amplify the Ae. aegypti sodium channel gene

\begin{tabular}{|c|c|c|c|c|c|}
\hline Section & Primer $\left(5^{\prime}-3^{\prime}\right)$ & Beginning nucleotide & End nucleotide & Length $(b p)$ & $\mathrm{Tm}$ \\
\hline \multirow[t]{2}{*}{ I } & GACAATGACCGAAGACTC & 17 & 693 & 677 & $46^{\circ} \mathrm{C}$ \\
\hline & ATGCTAATGCTATTACTACG & & & & \\
\hline \multirow[t]{2}{*}{$\|$} & GCGAGGTTTCATATTACAA & 614 & 1262 & 649 & $49^{\circ} \mathrm{C}$ \\
\hline & ACCCAAGAAGATAATCACAA & & & & \\
\hline \multirow[t]{2}{*}{ III } & CGTGGCACATGCTCTTCT & 1222 & 1833 & 612 & $55^{\circ} \mathrm{C}$ \\
\hline & TGACCGCGTTCGAGTCAT & & & & \\
\hline \multirow[t]{2}{*}{ IV } & CACAAGAACATTTGCCGTAC & 1792 & 2401 & 610 & $53^{\circ} \mathrm{C}$ \\
\hline & TCCTGGAACTTGAGCCAC & & & & \\
\hline \multirow[t]{2}{*}{ V } & GGCTCAAGTTCCAGGAGT & 2386 & 3012 & 627 & $51^{\circ} \mathrm{C}$ \\
\hline & CACCCACAAGCATACAATC & & & & \\
\hline \multirow[t]{2}{*}{ VI } & GTGGGATTGTATGCTTGTG & 2990 & 3592 & 603 & $52^{\circ} \mathrm{C}$ \\
\hline & ATGCCTCTATGATTCAGTTCGT & & & & \\
\hline \multirow[t]{2}{*}{ VII } & CCAAGGTGATAGGCAATT & 3520 & 4156 & 637 & $51^{\circ} \mathrm{C}$ \\
\hline & CAGGCGTTCGTAAAGTAAA & & & & \\
\hline \multirow[t]{2}{*}{ VIII } & CAAGTGGTTGGCGCTGGGTT & 4112 & 4768 & 657 & $63^{\circ} \mathrm{C}$ \\
\hline & CCGGCTITCTTCTTCTGTTCGT & & & & \\
\hline \multirow[t]{2}{*}{ IX } & TTCATCATCTTCGGGTCG & 4683 & 5326 & 644 & $52^{\circ} \mathrm{C}$ \\
\hline & AAGAACAGCAGCAGACAGA & & & & \\
\hline \multirow[t]{2}{*}{$x$} & GTCAAGGGTGCCAAAGGT & 5238 & 5914 & 677 & $55^{\circ} \mathrm{C}$ \\
\hline & TTCCGAGCGAAGAAGTCC & & & & \\
\hline \multirow[t]{2}{*}{$X \mid$} & ACATACCGATCTGTCGAG & 5830 & 6495 & 660 & $47^{\circ} \mathrm{C}$ \\
\hline & ATTTCTGTCGTGCTTCTG & & & & \\
\hline
\end{tabular}


DNA Extraction Kit according to the manufacturer's protocol (Takara, Japan). DNA was solubilized in a final volume of $100 \mu \mathrm{lddH} \mathrm{H}_{2} \mathrm{O}$.

\section{Allele-specific PCR (AS-PCR) genotyping of sodium channel genes}

AS-PCR was used to detect S989P, V1016G and F1534C mutations in each of the five wild Ae aegypti strains. A test using two PCR reactions for each individual was developed to diagnose each mutation (Fig. 1). The two reactions were exactly the same except that one contained a susceptible-specific primer and the other contained a mutant-specific primer. The outer primers and allele-specific inner primers for the three target mutations are listed in Table 2. A PCR diagnostic test was performed in accordance with the standard procedure with a total reaction volume of $25 \mu \mathrm{l}$, consisting of 80-100 ng genomic DNA, $14 \mathrm{mM}$ Tris- $\mathrm{HCl}, \mathrm{pH} 8.3$, $70 \mathrm{mM} \mathrm{KCl}, 4.5 \mathrm{mM} \mathrm{MgCl} 2,0.15 \mathrm{mM}$ each dNTP and $0.67 \mathrm{U}$ TakaRa rTaq. PCR conditions were one cycle of $94{ }^{\circ} \mathrm{C}$ for $3 \mathrm{~min}$, then 35 cycles of $94{ }^{\circ} \mathrm{C}$ for $30 \mathrm{~s}, 60$ or
$62{ }^{\circ} \mathrm{C}$ (60 ${ }^{\circ} \mathrm{C}$ for V1016G and F1534C, $62{ }^{\circ} \mathrm{C}$ for S989P) for $30 \mathrm{~s}$ and $72{ }^{\circ} \mathrm{C}$ for $1 \mathrm{~min}$, followed by one cycle of $72{ }^{\circ} \mathrm{C}$ for $7 \mathrm{~min}$. PCR products were checked by electrophoresis on $1.5 \%$ agarose gel in TAE buffer. Bands were visualized by ethidium bromide staining. The size of the PCR products for the detection of $k d r$ alleles were $240 \mathrm{bp}$ (S989P), 348 bp (V1016G) and 284 bp (F1534C), whereas the size of the products used as allele-nonspecific outer primers were 594 bp (S989P), 592 bp (V1016G) and 517 bp (F1534C).

\section{Correlation of resistance with the frequencies of different mutations}

In order to assess whether the frequency of different $k d r$ alleles in Ae. aegypti strains was related to resistance, the relationship between allele frequency and the $\mathrm{LC}_{50}$ values for cypermethrin and cyhalothrin was analysed with SAS Software 6.22 (SAS Institute Inc.). Coefficients of correlation were calculated between the frequency of different mutations in different strains and the corresponding $\mathrm{LC}_{50}$ values for each pyrethroid. To determine whether the
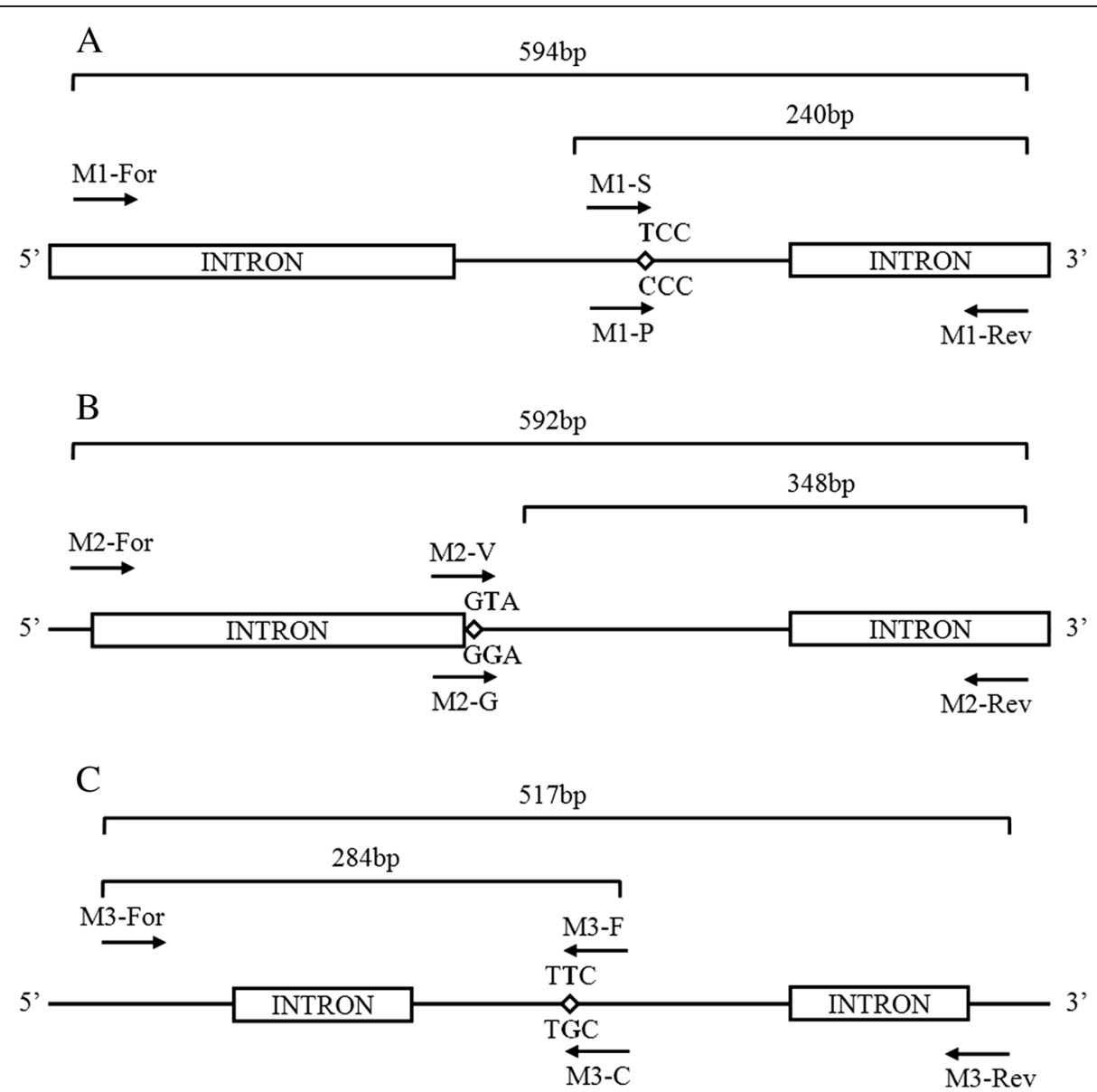

Fig. 1 Schematic diagram of AS-PCR test used to detect the S989P (a), V1016G (b) and F1534C (c) mutations in the sodium channel gene of Ae. aegypti 
Table 2 The specific primers used to amplify sodium channel gene mutations detected in Ae. aegypti

\begin{tabular}{lll}
\hline Mutation & Primers & Sequence (5'-3') \\
\hline S989P & M1-For & AATGATATTAACAAAATTGCGC \\
M2-Rev & GCACGCCTCTAATATTGATGC \\
M1-S & GCGGCGAGTGGATCGAAT \\
M1-P & GCGGCGAGTGGATCGAAC \\
V1016G & M2-For & GCCACCGTAGTGATAGGAAATC \\
& M2-Rev & CGGGTAAGTTCGTTAGTAGC \\
& M2-V & GTTCCCACTCGCACAGGT \\
M1534C & M2-G & GTITCCCACTCGCACAGGG \\
& M3-Rev & GGAGAACTACACGTGGGAGAAC \\
& M3-F & GCGTGAAGAACGACCCGA \\
M3-C & GCGTGAAGAACGACCCGC \\
\hline
\end{tabular}

correlation was significant, the null hypothesis of $r=0$, i.e. no correlation, was tested with a Student's $t$-test. Significance levels of these tests were adjusted using the Bonferroni Correction [26] to compensate for the increased likelihood of obtaining significant results by chance.

\section{Results}

\section{Correlation of resistance to different insecticides}

All wild strains had various levels of resistance to the insecticides tested (Table 3). The resistance ratio (RR) of each strain for cypermethrin and cyhalothrin ranged from 6.82 to 88.82 -fold and 4.82 to 88.87 -fold, respectively. The Munao strain had the highest resistance to these two pyrethroids. Resistance to cypermethrin and cyhalothrin was significantly correlated, i.e. strains with high resistance to cypermethrin tended to also be highly resistant to cyhalothrin $\left(R^{2}>0.9, P<0.05\right)$ (Fig. 2) which indicates cross-resistance to these two insecticides.

\section{Identification of sodium channel gene mutations}

Three mutations were identified from the cDNA of a pooled sample of $\mathrm{F}_{1}$ specimens of wild Ae. aegypti: S989P (TCC-CCC), V1016G (GTA-GGA), and F1534C (TTC-TGC), compared to the susceptible strain.

AS-PCR genotyping of mosquitoes carrying $k d r$ mutations After optimizing experimental conditions, AS-PCR could effectively distinguish individual mosquitoes that were homozygous or heterozygous for the S989P, V1016G and F1534C mutations (Fig. 3). All five wild strains were tested and the mutation frequencies are summarized in Table 4. The Qishui and Dongfang strains had no mutations at sites S989 and V1016, however, three strains from Ruili (Jinghan, Yundang and Munao) had high frequencies of these mutations which reached $100 \%$ in the Munao strain. At the F1534 locus, the opposite situation was apparent; mutation frequency was $100 \%$ in the Qishui strain but very low in the three Ruili strains, indeed, there were no mutations at this locus in the Munao strain. The Jinghan and Yundang strains carried all three mutations.

\section{Correlation between insecticide resistance and mutation frequency}

A significant positive correlation between the frequency of the S989P and V1016G point mutations and resistance to cypermethrin and cyhalothrin was observed when comparing $\mathrm{LC}_{50}$ values and the frequency of these sodium channel gene mutations (Fig. 4). However, the frequency

Table 3 Levels of cypermethrin and cyhalothrin resistance in different strains of Ae. aegypti

\begin{tabular}{|c|c|c|c|c|}
\hline Insecticide & Strain & $\mathrm{LC}_{50}(\mathrm{ppm})(95 \% \mathrm{CL})$ & $\mathrm{RR}^{\mathrm{a}}$ & Regression equations \\
\hline \multirow[t]{6}{*}{ Cypermethrin } & SS & $0.00093(0.00086-0.00100)$ & 1 & $Y=12.04407+3.97312 X$ \\
\hline & QS & $0.00663(0.00628-0.00701)$ & 7.13 & $Y=13.24042+6.07821 X$ \\
\hline & DF & $0.00634(0.00490-0.00927)$ & 6.82 & $Y=5.75003+2.61641 X$ \\
\hline & $J H$ & $0.03349(0.02218-0.06264)$ & 36.01 & $Y=2.77811+1.88336 X$ \\
\hline & YD & $0.02558(0.02303-0.02834)$ & 27.51 & $Y=3.86610+2.42842 X$ \\
\hline & MN & $0.08260(0.07227-0.09338)$ & 88.82 & $Y=2.45754+2.26918 X$ \\
\hline \multirow[t]{6}{*}{ Cyhalothrin } & SS & $0.00091(0.00079-0.00105)$ & 1 & $Y=7.75649+2.55102 X$ \\
\hline & QS & $0.00568(0.00531-0.00607)$ & 6.24 & $Y=9.59773+4.27341 X$ \\
\hline & DF & $0.00439(0.00382-0.00505)$ & 4.82 & $Y=7.39856+3.13863 X$ \\
\hline & $J H$ & $0.03879(0.03518-0.04299)$ & 42.63 & $Y=4.90089+3.47272 X$ \\
\hline & YD & $0.02794(0.02530-0.03092)$ & 30.70 & $Y=3.79551+2.44265 X$ \\
\hline & MN & 0.08087 (0.06909-0.09386) & 88.87 & $Y=2.59628+2.37709 X$ \\
\hline
\end{tabular}

SS susceptible strain, QS Qishui strain, DF Dongfang strain, JH Jinghan strain, YD Yundang strain, MN Munao strain

${ }^{a} \mathrm{RR}=$ resistance ratio. When the non-insecticide control was used, the percentage of natural mortality is $2.2 \%, 1.1 \%, 0 \%, 1.1 \%, 0 \%$ and $0 \%$ for $\mathrm{SS}, \mathrm{QS}, \mathrm{DF}$, JH, YD and MN strain 

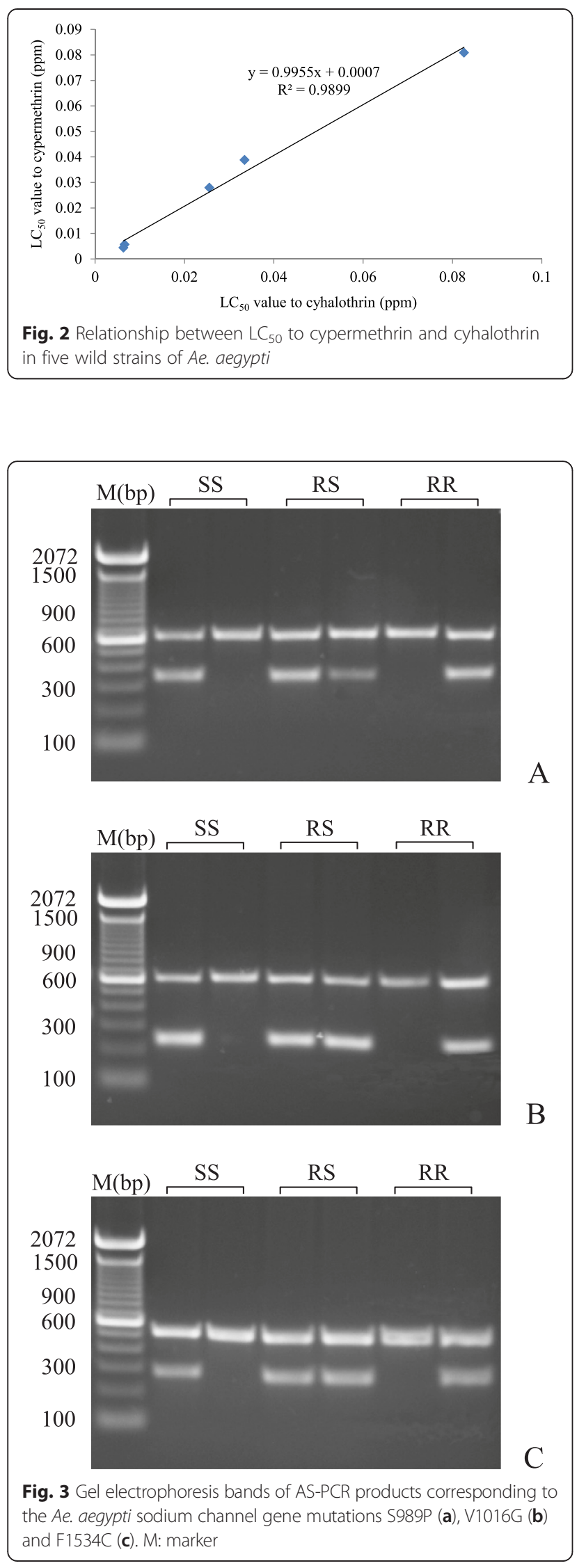

Table 4 Frequency of the S989P, V1016G and F1534C mutations in the sodium channel gene of five wild strains of Ae. aegypti

\begin{tabular}{llllll}
\hline Mutation & Strain & \multicolumn{4}{l}{ Genotype frequency (\%) } \\
\cline { 3 - 6 } & & SS & RS & RR & R \\
\hline S989P & QS & 100.0 & 0.0 & 0.0 & 0.0 \\
& DF & 100.0 & 0.0 & 0.0 & 0.0 \\
& JH & 2.8 & 30.6 & 66.7 & 81.9 \\
& YD & 0.0 & 33.3 & 66.7 & 83.3 \\
& MN & 0.0 & 0.0 & 100.0 & 100.0 \\
V1016G & QS & 100.0 & 0.0 & 0.0 & 0.0 \\
& DF & 100.0 & 0.0 & 0.0 & 0.0 \\
& JH & 0.0 & 16.7 & 83.3 & 91.7 \\
& YD & 0.0 & 9.1 & 90.9 & 95.5 \\
& MN & 0.0 & 0.0 & 100.0 & 100.0 \\
F1534C & QS & 0.0 & 0.0 & 100.0 & 100.0 \\
& DF & 3.0 & 18.2 & 78.8 & 87.9 \\
& JH & 84.8 & 15.2 & 0.0 & 7.6 \\
& YD & 90.9 & 9.1 & 0.0 & 4.6 \\
& MN & 100.0 & 0.0 & 0.0 & 0.0
\end{tabular}

SS: susceptible homozygote. SS $\%=S S /(S S+R S+R R) \times 100 \%$

RS: mutant heterozygote. RS $\%=R S /(S S+R S+R R) \times 100 \%$

RR: mutant homozygote. RR\% $=\mathrm{RR} /(\mathrm{SS}+\mathrm{RS}+\mathrm{RR}) \times 100 \%$

$\mathrm{R} \%=\mathrm{RR} \%+0.5 \times \mathrm{RS} \%$

QS Qishui strain, DF Dongfang strain, JH Jinghan strain, $Y D$ Yundang strain,

MN Munao strain

of the F1534C point mutation was negatively correlated with resistance to these two pyrethroids (Fig. 4).

\section{Correlation between the S989P and V1016G mutations}

The results show a strong linear relationship between the frequency of the S989P and V1016G mutations (Fig. 5). Co-occurrence of the S989P and V1016G mutations in five wild strains are presented (Table 5). The Qishui and Dongfang strains were all SS homozygotes and the Munao strain were all RR homozygotes, but the Jinghan and Yundang strains were either RS heterozygotes (13.9\% and $9.1 \%$ ) or RR homozygotes (66.7\%). In the remaining small proportion of individual mosquitoes, if the S989P mutation was detected as SS, the V1016G mutation was expressed as RS (2.8 \% in Jinghan strain). Whereas, if the S989P mutation was expressed as RS, the V1016G mutation was expressed as RR (16.7 \% in Jinghan strain, $24.2 \%$ in Yundang strain).

\section{Discussion}

Ae. aegypti is an important vector of dengue fever in China. It was previously thought that this species was only found in China south of latitude $22^{\circ} \mathrm{N}$. Climate change, the rapid development of tourism and transportation and 

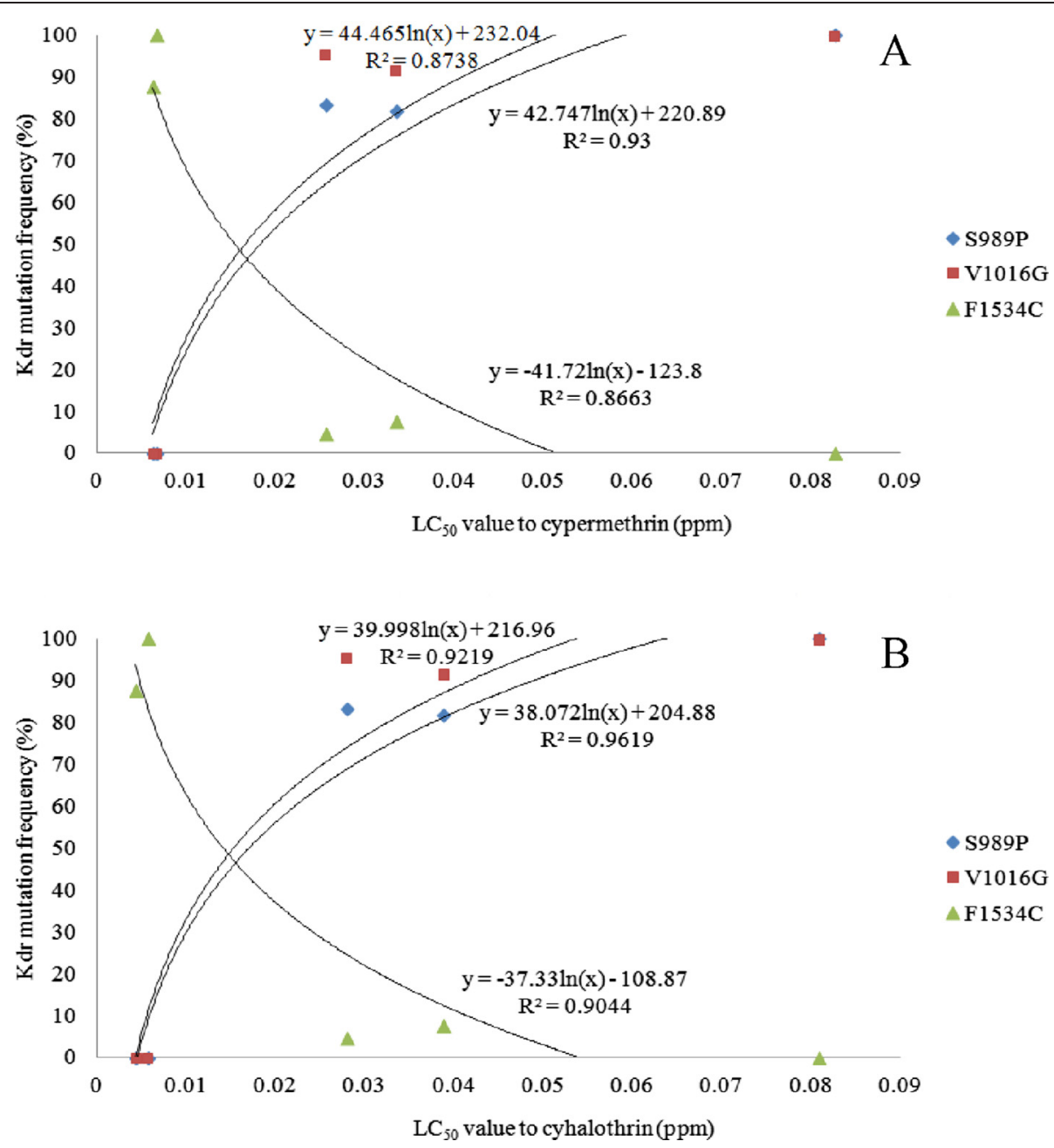

Fig. 4 Relationship between frequency of the S989P ( $)$, V1016G (- $)$ and F1534C ( $\mathbf{\Delta})$ kdr mutations and LC 50 to cypermethrin (a) and cyhalothrin (b)

increasing urbanization have resulted in an increase in Ae. aegypti breeding sites and consequently a marked change in the species' range in China. In recent years, the range of Ae. aegypti has expanded to approximately latitude $25^{\circ} \mathrm{N}$ [27].
The research presented in this paper is the first study of $k d r$ resistance mechanisms in wild Ae. aegypti strains in China. We used the bioassay recommended by the WHO to determine the level of pyrethroid resistance of five wild strains of Ae. aegypti. The results show that the

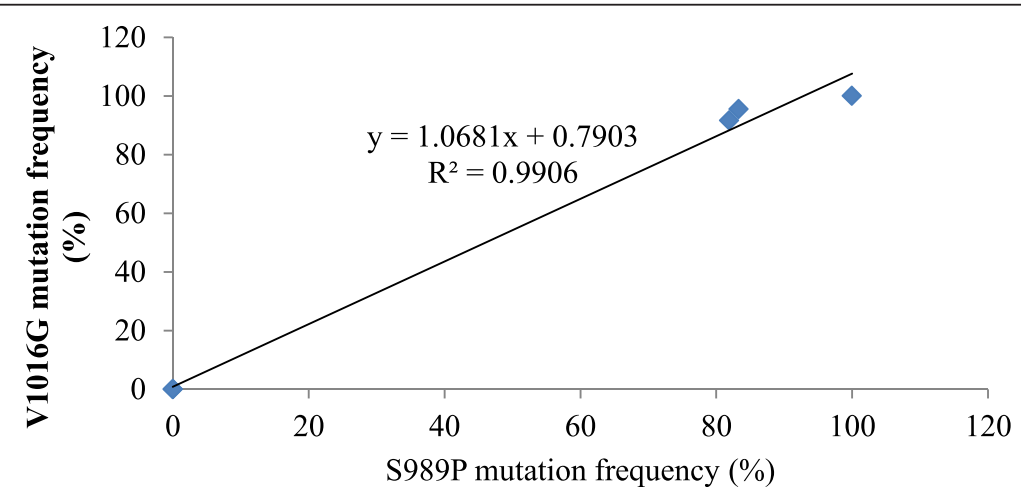

Fig. 5 Correlation between the frequency of the S989P and V1016G mutations 
Table 5 Co-occurrence of the S989P and V1016G mutations in five wild strains of Ae. aegypti

\begin{tabular}{|c|c|c|c|c|c|c|c|c|c|c|}
\hline \multirow[t]{2}{*}{ Mutation } & \multirow[t]{2}{*}{ Strain } & \multicolumn{9}{|c|}{ Genotype frequency (\%) } \\
\hline & & $\begin{array}{l}\text { S/S989+ } \\
\text { V/N1016 }\end{array}$ & $\begin{array}{l}\text { S/P989+ } \\
\text { V/N1016 }\end{array}$ & $\begin{array}{l}\text { P/P989+ } \\
\text { V/V1016 }\end{array}$ & $\begin{array}{l}\text { S/S989+ } \\
\text { V/G1016 }\end{array}$ & $\begin{array}{l}\text { S/P989+ } \\
\text { V/G1016 }\end{array}$ & $\begin{array}{l}\text { P/P989+ } \\
\text { V/G1016 }\end{array}$ & $\begin{array}{l}\text { S/S989+ } \\
\text { G/G1016 }\end{array}$ & $\begin{array}{l}\text { S/P989+ } \\
\text { G/G1016 }\end{array}$ & $\begin{array}{l}\text { P/P989+ } \\
\text { G/G1016 } \\
\end{array}$ \\
\hline \multirow[t]{5}{*}{ S989P + V1016G } & QS & 100.0 & 0.0 & 0.0 & 0.0 & 0.0 & 0.0 & 0.0 & 0.0 & 0.0 \\
\hline & DF & 100.0 & 0.0 & 0.0 & 0.0 & 0.0 & 0.0 & 0.0 & 0.0 & 0.0 \\
\hline & $J H$ & 0.0 & 0.0 & 0.0 & 2.8 & 13.9 & 0.0 & 0.0 & 16.7 & 66.7 \\
\hline & YD & 0.0 & 0.0 & 0.0 & 0.0 & 9.1 & 0.0 & 0.0 & 24.2 & 66.7 \\
\hline & MN & 0.0 & 0.0 & 0.0 & 0.0 & 0.0 & 0.0 & 0.0 & 0.0 & 100.0 \\
\hline
\end{tabular}

three wild strains obtained from Ruili in Yunnan (the Jinghan, Yundang and Munao strains) generally had high pyrethroid resistance. Highest and lowest levels of resistance to cypermethrin were 88.82 and 27.51 times, respectively, that of the susceptible strain, and for cyhalothrin, 88.87 and 30.7 times that of the susceptible strain. These high levels of resistance are probably related to extensive pesticide use in the Ruili region. Although Ae. aegypti was only first detected breeding in Yunnan in 2006 [28], pyrethroid insecticides have been extensively used since its discovery for mosquito control. The resultant selection pressure appears to have produced high levels of resistance to pyrethroids; our results show that cypermethrin and cyhalothrin are already unsuitable for controlling Ae. aegypti in the Ruili region.

Ae. aegypti collected from the Qishui and Dongfang were 7.13 and 6.82 times as resistant to cypermethrin, and 6.24 and 4.82 times as resistant to cyhalothrin, as the susceptible strain, which were much lower as compared to those of the three Ruili strains. The relatively low pyrethroid resistance in these Ae. aegypti strains may be the result of limited pyrethroid use in this economically-indigent area.

Our results indicate a significant positive correlation between the frequency of the S989 and V1016 mutations and resistance to cypermethrin and cyhalothrin in the five Ae aegypti strains examined. As such, the higher the frequency of these mutations, the higher the level of resistance to cypermethrin and cyhalothrin one would expect. Lin et al. [29] found a correlation between the frequency of the V1016G $k d r$ mutation and resistance to transfluthrin, d-allethrin, metofluthrin, esbiothrin and prallethrin. Moreover, the S989P mutation often co-exists with V1016G in pyrethroid resistant strains of Ae. aegypti [30]. However, V1016G/S989P individuals are no more resistant to pyrethroids than those carrying just one of the mutations [31]. This suggests that S989P does not enhance the resistance conferred by the V1016G mutation. Du et al. [31] speculated that S989P could compensate for any reduction in fitness caused by the V1016G mutation. Hirata et al. [32] states that S989P alone did not alter deltamethrin sensitivity, but when combined with V1016G, mosquitoes were ten times more sensitive to deltamethrin. Kawada et al. [33] states that S989P has always been found with V1016G, but V1016G has been found alone. S989P has never been found alone. From our results, the frequency of occurrence of S989P and V1016G heterozygotes and homozygotes, we speculate that the V1016G occurred before the S989P mutation.

Hu et al. [34] previously confirmed by the insertion of the $1534 \mathrm{C}$ equivalent mutation into the sodium channel gene of the cockroach that the F1534C mutation confers sodium channel resistance to type I, but not type II, pyrethroids. The $\mathrm{F}$ to $\mathrm{C}$ mutation drastically reduced sodium channel sensitivity to three type I pyrethroids; permethrin, NRDC 157 (a deltamethrin analogue lacking the a-cyano group) and bioresemthrin, but not to three type II pyrethroids (cypermethrin, deltamethrin and cyhalothrin). We found that the F1534C mutation was negatively correlated with resistance to cypermethrin and cyhalothrin, which suggests that the higher the frequency of this mutation in mosquito strains, the lower their resistance to these two insecticides; thus, the presence of this mutation not only does not enhance resistance to cypermethrin and cyhalothrin but in fact reduces it.

Research on Ae. aegypti in Thailand found, regardless of insecticide exposure status, that no homozygous 1016G mutants expressed the homozygous form of the 1534C mutation [35]. Mosquitoes with the homozygous 1534C mutation are more susceptible to deltamethrin than those heterozygous and homozygous for 1534 F [35]. This is a very interesting result which suggests that the F1534C mutation could potentially compensate for any reduction in fitness caused by the S989P and V1016G mutations.

\section{Conclusions}

The methods we established proved to be highly reliable and should aid future studies aimed at further determining the extent of $k d r$ mutations worldwide. The frequency of the S989P, V1016G and F1534C mutations were correlated with resistance to cypermethrin and cyhalothrin, and all three mutations were found to be common in $A e$. aegypti strains throughout Southern China. Our results also provide the first evidence of the S989P, V1016G and F1534C mutations in Ae. aegypti in Southern China, thereby increasing the knowledge of these mutations. The 
V1016G mutation appears to be the most important mutation in Ae. aegypti strains in Southern China at the present time, however, there exists the possibility that other mechanisms were also present and acting to confer resistance.

\section{Competing interests}

The authors declare that they have no competing interests.

\section{Authors' contributions}

CXL and TYZ jointly designed and coordinated the study, with the contribution of PEK and RDX. CXL drafted the article. MHZ and GW collected samples from different sites. TY, XXG, YMZ, YDD, DX and HDZ carried out the laboratory work and performed the statistical analysis. All authors read and approved the final manuscript submitted.

\section{Acknowledgments}

We would like to thank Lois Wood, Mark Halvorsen and Chris Holderman for assistance with laboratory procedures conducted in the University of Florida, U.S.A. This work was funded by grants from the Infective Diseases Prevention and Cure Project of National Ministry of Public Health of China (No. 2012ZX10004219).

\section{Author details}

${ }^{1}$ Department of Vector Biology and Control, State Key Laboratory of Pathogen and Biosecurity, Institute of Microbiology and Epidemiology, Beijing 100071, China. ${ }^{2}$ Entomology \& Nematology Department, University of Florida, Gainesville, FL 32611, USA. ${ }^{3}$ Anastasia Mosquito Control District, 500 Old Beach Road, St. Augustine, FL, USA.

Received: 19 September 2014 Accepted: 3 June 2015

\section{Published online: 12 June 2015}

\section{References}

1. Gubler DJ. Dengue and dengue hemorrhagic fever. Clin Microbiol Rev. 1998;11:480-96.

2. Townson $H$, Nathan MB, Zaim M, Guillet $P$, Manga $L$, Bos $R$, et al. Exploiting the potential of vector control for disease prevention. Bull World Health Organ. 2005:83:942-7.

3. Rodriguez MM, Bisset J, de Fernandez DM, Lauza' $n$ L, Soca A. Detection of insecticide resistance in Aedes aegypti (Diptera: Culicidae) from Cuba and Venezuela. J Med Entomol. 2001;38:623-8.

4. Lin YH, Teng HJ, Ho CM, Pai HH, Wu SC, Hsu EL. Insecticide resistance in Aedes aegypti during dengue epidemics in Taiwan. Formosan Entomol. 2003;23:263-74.

5. Narahashi T. Neuronal ion channels as the target sites of insecticides. Parmacol Toxicol. 1996;78:1-14.

6. Martinez-Torres D, Chandre F, Williamson MS, Darriet F, Berge JB, Devonshire AL, et al. Molecular characterization of pyrethroid knockdown resistance $(\mathrm{kdr})$ in the major malaria vector Anopheles gambiae s.s. Insect Mol Biol. 1998;7:179-84.

7. Enayati AA, Vatandoost $H$, Ladonni H, Townson $H$, Hemingway J. Molecular evidence for a kdr-like pyrethroid resistance mechanism in the malaria vector mosquito Anopheles stephensi. Med Vet Entomol. 2003;17:138-44.

8. Martinez-Torres D, Chevillon C, Brun-Barale A, Berge JB, Pasteur N, Pauron D. Voltage-dependent $\mathrm{Na}+$ channels in pyrethroid-resistant Culex pipiens $\mathrm{L}$ mosquitoes. Pest Sci. 1999;55:1012-20.

9. Brengues C, Hawkes NJ, Chandre F, McCarroll L, Duchon S, Guillet P, et al. Pyrethroid and DDT cross-resistance in Aedes aegypti is correlated with novel mutations in the voltage-gated sodium channel gene. Med Vet Entomol. 2003;17:87-94.

10. Ingles PJ, Adams PM, Knipple DC, Soderlund DM. Characterization of voltage-sensitive sodium channel gene coding sequences from insecticide susceptible and knockdown-resistant house fly strains. Insect Biochem Mol Biol. 1996;26:319-26.

11. Pinto J, Lynd A, Elissa N, Donnelly MJ, Costa C, Gentile G, et al. Co-occurrence of East and West African kdr mutations suggests high levels of resistance to pyrethroid insecticides in Anopheles gambiae from Libreville, Gabon. Med Vet Entomol. 2006;20:27-32.
12. Chen L, Zhong D, Zhang D, Shi L, Zhou G, Gong M, et al. Molecular ecology of pyrethroid knockdown resistance in Culex pipiens pallens mosquitoes. PLOS ONE. 2010;5:e11681.

13. Park Y, Taylor MFJ. A novel mutation L1029H in sodium channel gene hscp associated with pyrethroid resistance for Heliothis virescens (Lepidoptera: Noctuidae). Insect Biochem Mol Biol. 1997;27:9-13.

14. Saavedra-Rodriguez K, Urdaneta-Marquez L, Rajatileka S, Moulton M, Flores AE, Fernandez-Salas I, et al. A mutation in the voltage-gated sodium channel gene associated with pyrethroid resistance in Latin American Aedes aegypti. Insect Mol Biol. 2007;16:785-98.

15. Kawada H, Higa Y, Komagata O, Kasai S, Tomita T, Thi Yen N, et al. Widespread distribution of a newly found point mutation in voltage-gated sodium channel in pyrethroid-resistant Aedes aegypti populations in Vietnam. PLoS Negl Trop Dis. 2009;3:e527.

16. Martins AJ, Lima JB, Peixoto AA, Valle D. Frequency of Val1016lle mutation in the voltage-gated sodium channel gene of Aedes aegypti Brazilian populations. Trop Med Int Health. 2009;14:1351-5.

17. Harris AF, Rajatileka S, Ranson H. Pyrethroid resistance in Aedes aegypti from Grand Cayman. Am Trop Med Hyg. 2010;83:277-84.

18. Cui F, Raymond M, Qiao CL. Insecticide resistance in vector mosquitoes in China. Pest Manag Sci. 2006;62:1013-22.

19. Zeng LH, Sun DW, Zhao W, Li SG, Yang X. Determination of the susceptibility of Aedes aegypti and Aedes albopictus to commonly used insecticides in Hainan province. Chin J Vector Biol Control. 2010;21:148-9.

20. WHO [World Health Organization]. Report of the WHO Informal Consultation on the evaluation and testing of insecticides. Geneva, Switzerland: World Health Organization; 1996. CTD/WHO PES/IC/96.1.

21. Schoofs GM, Willhite CC. A probit analysis program for the personal computer. J Appl Toxicol. 1984;4:141-4.

22. Orshan L, Kelbert M, Pener H. Patterns of insecticide resistance in larval Culex pipiens populations in Israel: dynamics and trends. J Vector Ecol. 2005;30:289-94.

23. Aiyar $\mathrm{A}$. The use of CLUSTAL $W$ and CLUSTAL $X$ for multiple sequence alignment. Methods Mol Biol. 2000;132:221-41.

24. DNAstar. (http://www.dnastar.com/t-allproducts.aspx)

25. Tompson JD, Higgins DG, Gibson TJ. Clustal-W-improving the sensitivity of progressive multiple sequence alignment through sequence weighting, position-specific gap penalities and weight matrix choice. Nucleic Acids Res. 1994:22:4673-80

26. Rice WR. Analysing tables of statistical tests. Evolution. 1989;43:223-5.

27. Xie $\mathrm{H}$, Zhou HN, Yang YM. Advances in the research on the primary dengue vector Aedes aegypti in China. Chin J Vector Biol Control. 2011;22:194-7.

28. Wang PY, Zhou HN, Wu C, Gu YA, Dong LM, Zhu GJ, et al. Investigation of dengue vector Aedes aegypti in Yunnan province. Chin J Vector Biol Control. 2006;17:507-8

29. Lin YH, Tsen WL, Tien NY, Luo YP. Biochemical and molecular analyses to determine pyrethroid resistance in Aedes aegypti. Pestic Biochem Physiol. 2013;107:266-76.

30. Rinkevich FD, Du Y, Dong K. Diversity and convergence of sodium channel mutations involved in resistance to pyrethroids. Pestic Biochem Physiol. 2013;106:93-100.

31. Du Y, Nomura Y, Satar G, Hu Z, Nauen R, He SY, et al. Molecular evidence for dual pyrethroid-receptor sites on a mosquito sodium channel. Proc Natl Acad Sci U S A. 2013;110:11785-90.

32. Hirata K, Komagata O, Itokawa K, Yamamoto A, Tomita T, Kasai S. A single crossing-over event in voltage-sensitive $\mathrm{Na}+$ channel genes may cause critical failure of Dengue mosquito control by insecticides. PLoS Negl Trop Dis. 2014;8:e3085.

33. Kawada H, Oo SZM, Thaung S, Kawashima E, Maung YNM, Thu HM, et al. Co-occurrence of point mutations in the voltage-gated sodium channel of pyrethroid-resistant Aedes aegypti populations in Myanmar. PLoS Negl Trop Dis. 2014:8:e3032.

34. Hu Z, Du Y, Nomura Y, Dong K. A sodium channel mutation identified in Aedes aegypti selectively reduces cockroach sodium channel sensitivity to type I, but not type II pyrethroids. Insect Biochem Mol Biol. 2011;41:9-13.

35. Stenhouse SA, Plernsub S, Yanola J, Lumjuan N, Dantrakool A, Choochote W, et al. Detection of the V1016G mutation in the voltage-gated sodium channel gene of Aedes aegypti (Diptera: Culicidae) by allele-specific PCR assay, and its distribution and effect on deltamethrin resistance in Thailand. Parasit Vectors. 2013;6:253. 